\title{
Customer-Engineer Relationship Management for Converged Communications Service Providers
}

\author{
H Lee, P Mihailescu, J W Shepherdson, M A Silburn, Y Z Wei
}

\begin{abstract}
Thanks to the advent of converged communications services (often referred to as 'triple play'), the next generation Service Engineer will need radically different skills, processes and tools from today's counterpart. Why? in order to meet the challenges of installing and maintaining services based on multi-vendor software and hardware components in an IP-based network environment. The converged services environment is likely to be 'smart' and support flexible and dynamic interoperability between appliances and computing devices. These radical changes in the working environment will inevitably force managers to rethink the role of Service Engineers in relation to customer relationship management. This paper aims to identify requirements for an information system to support converged communications service engineers with regard to customer-engineer relationship management. Furthermore, an architecture for such a system is proposed and how it meets these requirements is discussed.
\end{abstract}

\section{Introduction}

Engineering field-force management is growing in importance as the mobile workforce is one of the main channels for detecting the changing needs of Customers and obtaining feedback on new products and services. This leads us to propose the concept of Customer-Engineer Relationship Management (CeRM), which we define as all Customer Relationship Management (CRM) [5] activities utilising Service Engineers. [5] defines CRM as "a comprehensive strategy and process of acquiring, retaining, and partnering with selective Customers to create superior value for the company and the Customer. It involves the integration of marketing, sales, Customer service, and the supply-chain functions of the organization to achieve greater efficiencies and effectiveness in delivering Customer value." From this definition, CeRM can be considered as a subset of those CRM activities which mainly concentrate on "retaining and partnering with selective Customers" [5] by utilising Service Engineers.

The recent trend of telecommunications companies transforming themselves into converged communications (triple play - telephony, internet and digital media) service providers has revealed that their Service Engineers need to display more CRM skills, as their behaviour strongly affects Customer satisfaction. Also, the information systems that support these 'new wave' Service Engineers must provide different services and functionalities from those offered by today's Service Engineer support solutions.

This paper proposes an architecture for a system to support CeRM activities, in the context of the provision and repair of converged communications services. Section 2 discusses other work related to CeRM and CRM. Important issues are identified in Section 3, and the way in which they are addressed by our architecture is covered in Section 4. Finally, section 5 summarises the paper and details our future research direction with regard to the implementation of a CeRM system.

\section{Related Work}

The research on support systems for Service Engineers can be classified into three categories: job scheduling [5], human-computer interface issues [5], and design of mobile task support systems [5]. This section introduces our research efforts on mobile task support systems.

We have been conducting investigations into the use of mobile devices (such as Personal Digital Assistants) since our involvement in the EU IST Project LEAP: $A$ Lightweight Extensible Agent Platform [5], which closed in mid-2002. A number of advanced prototypes that explore options for individual and team-based approaches to receiving, progressing and closing jobs in a Mobile Work environment have been built and evaluated. This has included internal trials with Wholesale Survey Officers, Payphones Engineers and Retail Field Service Engineers, as well as the TeamWorker prototype [5].

The trial [5] with BT Wholesale Survey Officers was based on an iPAQ PDA (with an external GPRS modem and an external GPS receiver). The features of the trial system included always-on, wireless access to personal job queues on a backend job allocation system; monitoring users' job queues and automatically alerting them to the arrival of new jobs; pannable maps centred on the job location with optional BT Network overlays; the use of GPS to provide features such as 'find my nearest petrol station' and 'find colleague closest to a given job location'. The latter was used in conjunction with a job trading system that allowed a User to offer a job they were unlikely 
to be able to start on time to the colleague closest to the job location. The demonstrable business benefits were reduction of travel time and increased responsiveness to Customers in the form of reduced waiting times.

On the other hand the trial with BT Payphones Engineers [5] used the Panasonic P2 (a semi-ruggedised PDA with built-in phone, GPRS, WiFi and Bluetooth connectivity). The application featured always-on, wireless access to shared job queues on a backend work allocation system; the ability to reserve and un-reserve jobs; partial job closure; automatic notification of the arrival of new jobs with a given importance score; hands-free operation (text to speech for incoming messages, automatic speech recognition for command-word recognition); route planning via the use of a GPS receiver and an integrated Satellite Navigation package. The trial showed a number of business benefits, as the highest value jobs got immediate attention and route planning reduced travelling times. Compared with the previous trial, it also served to highlight the speed at which mobile computing devices are evolving.

The TeamWorker prototype focussed on Team working/coordination, work visibility and work flexibility, as explained below.

- Team working/coordination: engineers typically work alone, and have little or no formal interaction with other team members. TeamWorker addressed this issue by providing a set of coordination services such as job trading, job assistance, and organizing meetings (work or social) that enabled engineers to interact with each other in real time;

- Work visibility: As TeamWorker provided wireless communication over a GPRS network, engineers benefited from the always-on aspects. Therefore, engineers not only had the ability to access the latest job information, including any changes to their schedules (which were automatically pushed out to them), but could update their progress in real time. In addition, management had greater visibility as to what was occurring in the field, and could respond to changes more quickly;

- Work flexibility: TeamWorker was developed for use with mobile computing devices, so engineers could take the device with them regardless of whether they were at a customer's premise, or up a pole. This not only enabled engineers to access the system at any time and any place, but also increased the ability of other team members or management to get in touch with them.

\section{CeRM Challenges for Converged Communications Service Providers}

The role of Telecommunications Service Engineers is evolving from 'command and control' operatives to front line company ambassadors. The three goals related to CeRM are: acquire Customer, retain Customer, and explore sales opportunities. They can be achieved by executing the general CRM activities as shown in Fig 1, which also shows how Service Engineers can contribute.

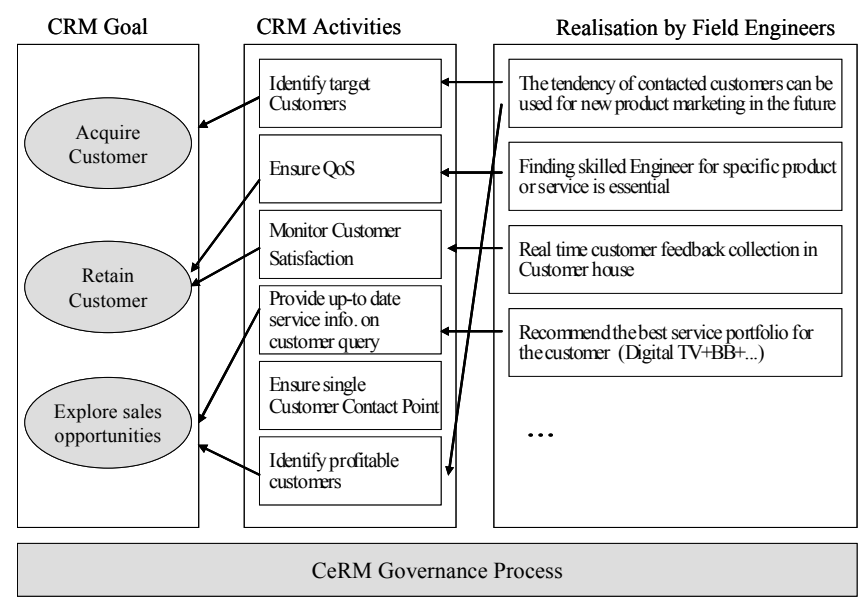

\section{Fig $1 \quad$ CeRM Model}

The plethora of hardware and software combinations which will be found within Customers' premises [5] in the next three to five years will present many problems for Service Engineers, unless appropriate technology is made available to extract, integrate and present information to them, in order to reduce information overload.

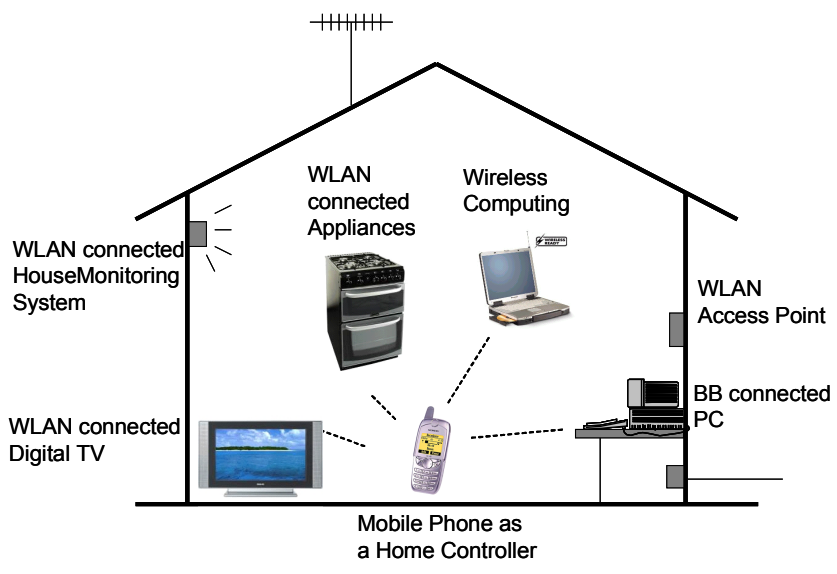

Fig 2 Customers with Converged Communications Services

Fig 2 shows what could be a typical working environment for next generation services engineers. Converged services will allow Customers to inhabit a "Digital Home" wherein domestic appliances, telecommunication devices, and computing devices can communicate with each other, and family members can (remotely) monitor the state of their home via various mobile devices. The sheer complexity of connecting and maintaining disparate personal computers, firewalls, set-top boxes, wireless networks and other peripheral devices, suggests to us that the following four areas need to be considered in the design of information systems to support 
CeRM activities undertaken by converged communications service providers:

- Knowledge Support for Converged Service Engineers;

- $\quad$ Organising Converged Service Engineers;

- Integration of Heterogeneous Systems;

- Security (especially trust and reputation issues).

\subsection{Knowledge Support for Converged Service Engineers}

The diversity of tasks undertaken by the Converged Service Engineer is much greater, and involves more technical complexity than maintaining Plain Old Telecommunications Services. This forces the communications service provider to organise its field force with diverse skill sets with regard to Appliances, Computing devices and/or Telecommunication equipment and so on. The major challenges in the implementation of CeRM are mainly focused on empowering Converged Service Engineers so that they can perform jobs on Customers' premises, deal with any Customer queries and so on.

\subsection{Organising Converged Service Engineers}

Due to the diversity on mobile tasks, it may not be possible for a converged communications service provider to have fully qualified Converged Service Engineers who can deal with all the Customer queries or provide installation or repair services for the full range of communications services offered. The main issue in this area is how to organise Converged Service Engineers so the company has the flexibility to respond to these new Customer service challenges.

\subsection{Integration of Heterogeneous Systems}

The sheer diversity of communications services in a single Customer location inevitably requires integration with external partners. This means an information system to support CeRM activity should allow Converged Service Engineers to have access to heterogeneous external partner systems according to predefined business rules.

\subsection{Security}

Integration with heterogeneous internal and external systems naturally causes a security issue. That is, there should be a validation process for any information collected via an external partner's Intranet or the Internet. Furthermore, communications among various components of an information system to support CeRM should be based on a security assured platform.

\section{Customer-Engineer Relationship Management System}

Fig 3 shows the overall architecture of the proposed CeRM system, which is based on the principles identified in Section 3. In this architecture, a multi-agent system is employed as the distributed and collaborative computing technology. An Intelligent Agent on a mobile device collaborates with other Information Agents (or Wrapper Agents) to access heterogeneous knowledge sources (or legacy systems). The communication between different agents (from Personal Agent to Information Agent or Wrapper Agent, from Personal Agent to Personal Agent, from Information Agent to Wrapper Agent or vice versa) is performed via conversational components (C-COMs) [5]. A C-COM is a software component that an Agent can dynamically 'plug and play' in order to receive/provide new services from/to other Agents. The use of C-COMs facilitates the design of a service oriented architecture (SOA) [5]. The net effect of this is to give the Service Engineer access to information and tools necessary to diagnose, repair, configure and connect a multitude of components. This process could be initiated by the Service Engineer's mobile device once it detects a wireless access point on the Customer's premises.

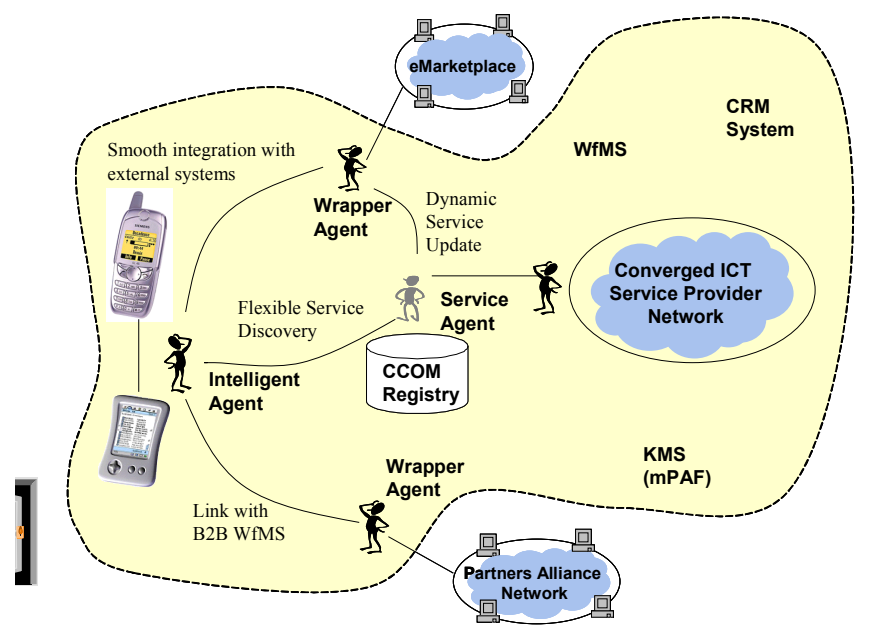

Fig 3 Overall architecture of CeRM system

In this architecture, a Service Agent records the details of all available services (such as Web or Agent Services) in a local Registry.

\subsection{Mobile Knowledge Management}

A typical service company holds a wealth of information for its Engineering workforce, ranging from health and safety guidelines to network routing maps. Unfortunately, a large amount of this information is often either in unstructured or semi-structured format, and is spread across multiple data sources. New data sources may appear which are not widely advertised within the Company, while others are no longer maintained and become obsolete. Typically this information is accessible via a basic search engine that only provides minimal data filtering features, thus returning multiple results (many of which are not directly relevant) that must be manually sifted.

Our approach to fulfilling the future knowledge management requirements of Converged Service Engineers is to leverage the features provided by already established 
knowledge management frameworks such as the Intelligent Personal Agent Framework [5] (iPAF). Through the use of the C-COM framework, we plan to expose these knowledge management services via new C-COMs that will be installed within an intermediate Agent (the wrapper agent) as shown in Fig 3. This wrapper Agent mediates messages from the Agents requesting knowledge management services (such as those running on a PDA), and the actual knowledge management service. Each new service provided by the wrapper Agent has a corresponding $\mathrm{C}-\mathrm{COM}$ which is registered with the $\mathrm{C}-\mathrm{COM}$ registry, thereby allowing other agents to access the services without worrying about the inner workings of the specific knowledge management system. This also enables us to swap one knowledge management system with another, without having to re-start the system.

We have identified three key knowledge management services that will help future Converged Service Engineers in their day to day activities. These are: 1) find relevant information, 2) find expert, and 3) update knowledge. Each of these three services is described below.

\section{Find Relevant Information}

With such a wide range of potential service offerings, it is envisaged that the future Converged Service Engineer will encounter may different scenarios during their day to day activities. One day she may be troubleshooting a faulty computer, the next day re-configuring an Internet-enabled domestic appliance. It will not be feasible to expect that an Engineer will have all the required knowledge or training to handle every possible scenario. Therefore Engineers will need to rely heavily on the knowledge accumulated within the Company when confronted with unfamiliar scenarios.

The 'find relevant information' service has been designed to address this need. This service will be both proactive and reactive, in that in the first instance, when an Engineer is given a job, it will automatically retrieve any information that has been linked with that job. For example, if a previous Engineer wrote a note about a fix they performed at the same premises this will be retrieved, or if a bulletin was issued on faulty equipment of the type that is present at the premises, this will also be made available.

An Engineer will be able to request additional information as and when needed, e.g. when they are trying to troubleshoot a problem. A diagnostic wizard will guide the Engineer through the process by providing them with only relevant options based initial on the type of job and location, and then by their selections. For example, if an Engineer is troubleshooting a faulty wireless network, she may be presented with an initial list of known wireless access products from which specific solutions will be presented based on the type of product selected. Finally the type of information retrieved will not be limited to just plain text, it could also be an image such as a network routing map, a document with embedded images, or an audio/video file.

\section{Find Expert}

As Engineers have different skills and abilities, they are more suited to handling different types of situations. For example, one Engineer may be highly skilled at troubleshooting Broadband issues, while another is skilled in PC diagnostics. The 'Find Expert' service allows Engineers to tap in to the available skills within the Company, by enabling them to get in touch with the right person based on the current problem scenario.

A wizard will be provided for the Engineer to enter the type of problem she is facing in order to determine who to contact. For example, an Engineer troubleshooting a faulty USB device will enter this fact, and the service will perform the matching process on their behalf to locate an appropriate person. Once the right person has been located, the Engineer will have the ability to communicate with them using a variety of communication mechanisms such as VoIP, GSM, text messaging, video etc. This type of service is also especially suited to supporting novice Engineers by helping them to get in touch with an experienced mentor when confronted by unfamiliar situations.

\section{Update Knowledge}

Over time Engineers will pick up new knowledge about e.g. a particular job, equipment type or job location that will be relevant and helpful for other Engineers. Therefore the update knowledge service allows Engineers to record this new knowledge so that it is accessible to others. Each new piece of information entered will be classified accordingly. For example, if an Engineer enters a 'hint' about the location of a piece of equipment within a Customer's premises, this will be associated with the location. Therefore any subsequent jobs based at this location will also reference this hint.

\subsection{Converged Service Engineer Organisation Model}

Studies have shown that "the average consumer will return the products to the store if he or she can not complete the setup of a new piece of electronic equipment within 15 to 30 minutes after he or she first takes it out of the box." [5]. Given an environment where Customers are connected to a high speed digital network, and use one or more converged services (see Fig 2), we surmise that the engineer of the future should display the following attributes:

- Has an Information Technology rather than Telecommunications background (i.e. little or no knowledge of the PSTN network);

- Is Customer focused (does everything possible, is not "pressed for time", is the "Customer's choice", explains everything);

- Shows flexibility (not rostered, works whenever needed, will do "extra" tasks, is highly mobile); 
- Is trustworthy (keeps customer records confidential and up-to-date, always recommends the right course of action);

- Behaves in a straightforward manner (communicates at customer's level, works with appropriate colleagues to get job done).

We envisage four scenarios for how a Converged Services Company might organise its Engineering workforce to provide support for Converged Service Customers as shown in Fig 4. These scenarios are not necessarily exclusive - there could be an element of mix and match, depending on various market conditions:

- Self-employed singleton digital plumbers, working on own account (c.f. plumber);

- Groups of employed/self-employed digital plumbers working as a disparate collective (c.f. taxi firm);

- Groups of employed/self-employed digital plumbers working as a co-operative (c.f. GP Surgery);

- $\quad$ IO - inside and outside team (c.f. current Customer Support Teams, handles the end to end connection as well as the customer premises equipment).

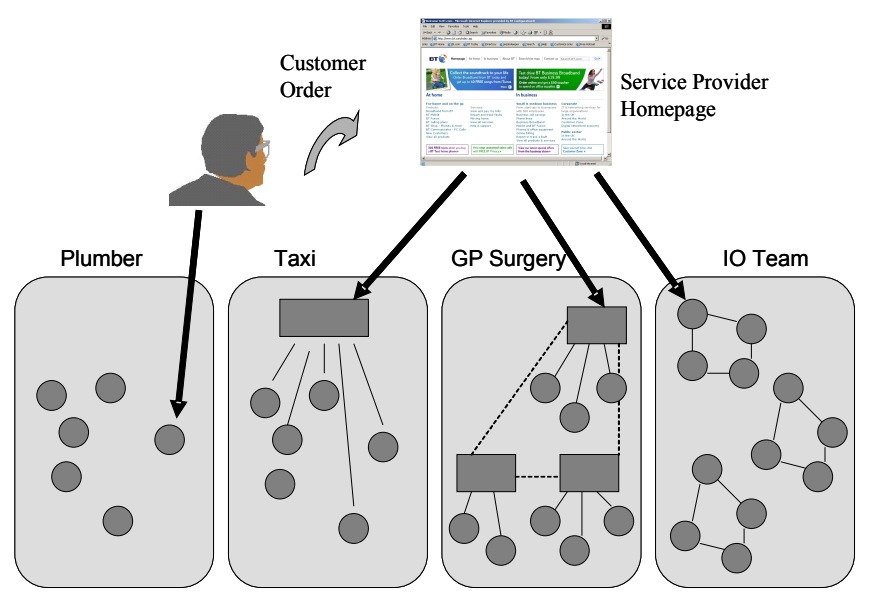

Fig 4 Organisational models

\subsection{Integration with Other Services}

In order to facilitate a CeRM system, integration with a number of other systems needs to take place. These other systems take a number of forms and their usage depends on the type of working style(s) implemented.

In order for the Engineer to fit new equipment or replacement parts these parts must first be obtained. While some small common parts may be carried by the Engineer for immediate replacement, higher value or specialist parts are ordered on demand by the Engineer, via a procurement system. To facilitate this, a number of sourcing arrangements must be in place and the Engineer must be provided with the item's cost and more importantly a delivery date in order for a new appointment to be made. This will become the eMarketplace [5] as shown in Fig 3. The eMarketplace will need to interact with many suppliers, manufacturers, also item and supplier reviews so that either a direct replacement or a suitable alternative can be obtained. For systems of this nature Nissen [5] suggests that "intelligent agents offer excellent potential and capability for supply chain management." It will be possible to provide the Engineer, and therefore the Customer, with a choice of solution based on a number of factors including cost, delivery time and product/vendor reputation with adaptable ratings for each. This may be influenced by any Service Level Agreements (SLA) or Call Off services in place, giving a higher weighting to those items or organisations. The eMarketplace will be accessed through a wrapper agent giving the eMarketplace the degree of separation necessary.

The second part of appointments story involves booking $3^{\text {rd }}$ party Engineers to attend. There may be many reasons for using $3^{\text {rd }}$ party Engineer, e.g. the warranty for a faulty item requires the use of the manufacturer's representative, or the problem requires a specialist in a particular technical area. To book appointments of this nature requires real-time integration with the booking systems of the $3^{\text {rd }}$ party organisation [5]. There may also be an opportunity to use a competitive sourcing model similar to the procurement model, where the system is able to recommend a particular engineer or organisation to attend based on availability, cost and/or reputation. This is the Partners Alliance Network shown in Fig 3 and is accessed through another wrapper agent.

Finally the details need to be fed back via the Service agent, ensuring that the Customer, billing and appointment details are recorded on the relevant systems.

We have looked at integration with two types of services, and a similar approach could be taken for other services. The flexible approach described above is needed in the fluid business world in which service providers operate to ensure that changes can be made simply, quickly and in a cost-effective manner.

There is a large volume of work being done in the eBusiness and Business to Business (B2B) space, and any CeRM system should tap into this work. Hu \& Grefen [5] state that "Though many business-to-business protocols are developed to support B2B interaction, none are generally accepted. A B2B system should support different B2B protocols dynamically to enable interaction between diverse enterprises." They go on to propose a componentbased architecture with the interfaces to the $3^{\text {rd }}$ party defined only at the very edge of the system, enabling all the internal messaging to be kept consistent. The type of integration proposed means that little of the application is affected when changes are made in the $3^{\text {rd }}$ party's systems, when new links with a company are forged or when an under-performing one is removed. 


\subsection{Security}

In the CeRM system that becomes more and more reliant on software entities (some provide/sell services whereas others consume services), security is an important concern. Here, we use the term 'software agent' in place of 'software entity', because a software agent can be viewed as a software entity that is situated in and interacts with its environment to achieve its goals. Without security management, an agent is not willing to share its private information or its services with other agents. In addition, security breaches can cost huge amounts of time and money. In this section, we identify the potential security concerns in our CeRM system and discuss some possible solutions.

In future communications services, many components may be widely distributed in a decentralised control regime. The open nature of the services undoubtedly presents a number of challenges. Suppose the intelligent agent located on the mobile device shown in Fig 3 requests a service on behalf of a user. The request is made through the service provider network that integrates offerings from service providers in a smooth and flexible manner. Because of the open nature of this network, service agents can join or leave at any time. It is conceivable that an agent could change its identity on re-joining and hence avoid punishment for any past wrong doing. Thus the system needs a mechanism to prevent such bad behaviour. On the other hand, different agents can provide the same service but with different cost or quality, so a consumer agent needs to reason about which service agent to interact with and to what level to trust it. Conversely, service agents also need mechanisms to evaluate the trustworthiness of consumer agents, as agents represent different stakeholders, each with their own aims and objectives. This means that all agents are self-interested and aim to maximize their own utility, which may be detrimental to their collaborators. Therefore, when building applications in the converged communications service provider's network, there is a need to design mechanisms that ensure all service agents gain equivalent utilities (or payments) for the services they have provided and all consumer agents gain equivalent services for the amount they have paid.

To date, there are many trust and reputation mechanisms developed to regulate agents' behaviour. In our context, what we need essentially is to build a protocol and a set of strategies. The protocol is a set of rules that drive all agents' behaviour and coordinate their interactions. The protocol should prevent agents from lying or manipulating each other through collusion so as to satisfy their selfish interests and hurt their collaborators. Having the protocol in place, on the other hand, by using suitable strategies, an agent should be able to reason which interactions are likely to gain maximal utility. Thus, we aim to classify the security issues in to two levels: the system protocol level and the individual strategy level. They are dealt with respectively.
To build trustworthy protocols, we need to consider the following issues. First, it is possible to build a protocol that makes lying or colluding agents gain no reward or receive punishment. Second, when two agents interact with each other, the protocol should be able to reveal one agent's reputation to the other. Knowing the collaborator's positive (or negative) reputation, an agent is able to reason and make decisions in future interactions with its collaborator. Third, a protocol should be able to manage new agents' indirect reputations. In case a new agent enters a system without any previous interactions with other agents, a protocol should try to provide proof of their reliability through references from trusted third parties.

With the protocol in place, an individual agent needs a set of strategies to reason and make decisions. From the point of view of the agent, it needs to consider the following issues to make rational decisions. On the one hand, an agent should be able to learn its collaborator agents' behaviour through interactions. Thus, the agent is able to identify which agent is the more (or less) reliable and develop strategies to deal with it in future interactions. On the other hand, the agent should also be able to solicit information concerning the reputation of its collaborator from other agents. In this way, an inexperienced agent is able to identify a reliable collaborator to interact with, if sufficient indirect information is obtained.

Having identified the main needs in building trustworthy protocols and strategies, we find that there are some other issues that need to be considered. First, context issues should be included. If a trustworthy agent provides a poor service because of its changing strategies with respect to its working environment, there should be environmental variables dealing with the unexpected decrement of its reputation. Second, we need consistent metrics for reputations. In some applications, different agents evaluate the quality of service with different metrics (e.g. different aspects of the service) when they request the same kind of service. This makes the reputation ratings for the same service agent from different consumer agents inconsistent. Therefore, such reputation ratings cannot deliver concrete trustworthiness. Last, social networks, i.e. groups of agents achieving their objectives simultaneously through their group interactions, are often assumed to exist in most security mechanisms. However, there is a lack of definition for the connections (or relationships) between agents. We believe well-defined relationships among agents within the social networks (e.g. partnerships in coalitions or memberships of the same organizations) will help achieve clearer evaluations of reputation and trust.

\section{Conclusions}

Converged Services Engineers will need radically different skills, processes and tools from today's counterpart, in order to meet the challenges of installing and maintaining triple play services. There are many ways in which the workforce can be organised and motivated in order to meet 
these challenges, and four possible models were described. Simulation work will be undertaken to examine the relative costs and benefits of each of these models.

We proposed a CeRM System architecture to support Field Engineers of the future converged communications service providers, and identified major issues that should be resolved by a CeRM system. The implementation of the architecture will be based on the authors' existing research experience with mobile task support systems, by integrating leading edge mobile computing, multi-agent systems and other emerging technologies. However, the CeRM system will also focus on:

- the use of Web-service and Semantic Web models for accessing, orchestrating and scheduling mobile user services and business processes;

- $\quad$ making these services context aware;

- $\quad$ and modelling cooperative work-flow for mobile users.

Finally, much more needs to be done to add context-based retrieval and blending of seemingly disparate information sources.

\section{Acknowledgements}

Much of the section on organisational models is based on a study conducted by Jon Malpass. The work described here was funded by BT Group CTO's Improving Customer Service \& Systems Venture - many thanks to Paul O'Brien for his advice and guidance.

\section{References}

1. Roseman $\mathbf{M}$ and Bassir $\mathrm{M}$ : 'Customer Relationship Management', http://www.leonardo.com.au/files/crme.pdf, SAPIENT College: Process Management (2000).

2. Parvatiyar A and Sheth JN, 'Customer Relationship Management: Emerging Practice, Process, and Discipline', Journal of Economic and Social Research, 3 No 2, pp 1 34 (2002).

3. Venkataramana V and Somayajulu G, 'Customer Relationship Management - A Key Success Factor For Telecom Sector', in Journal of Telecom Finance and Management, 51 No 1, pp 41 - 45 (2002).

4. Garwood GJ: 'Work Manager', BT Technol J 14, No 3 (1996).

5. Schmidt A, Lauff, M, and Beigl, M: 'Handheld CSCW', Proceeding of Workshop on Handheld CSCW at CSCW '98, Seattle (http://www.teco.edu/hcscw/papers.html) (1998).

6. Lee H, Mihailescu P, and Shepherdson JW: 'Multi-Agent Technology as an enabler of Computer Supported Cooperative Work for the Mobile Workforce', Lecture Notes in Artificial Intelligence (SCIE), 3191, pp 183 - 198 (2004).
7. Berger M, Buckland B, Bouzid M, Lee $\mathrm{H}$, Lhuillier $\mathrm{N}$, Olpp, D, Picault J, and Shepherdson JW: 'An Approach to Agent-based Service Composition and it's Application to Mobile Business Processes', IEEE Transactions on Mobile Computing (SCIE), 2 No 3, pp 197 - 206 (2003).

8. Lee H, Mihailescu P, and Shepherdson JW: 'TeamWorker: An Agent-based Support System for Mobile Task Execution', Whitestein Series in Software Agent Technologies - Applications and Prototypes II, edited by M. Calisti, M. Klusch and R. Unland, (2005).

9. Lee H, Buckland M and Shepherdson JW: 'A multi-agent system to support location-based group decision making in mobile teams', BT Technol J, 21 No 1 (2003).

10. 'ERIC takes the rough with the smooth', BT Today, July (2005).

11. Harrison M, Bull P, 'Managing broadband home networks', BT Technol J, 24 No 1, pp 79 - 85 (2006).

12. Lee $H$, Mihailescu $P$, and Shepherdson JW: 'Conversational Component-based Open Multi-agent Architecture for Flexible Information Trade', Lecture Notes in Artificial Intelligence (SCIE), No 2782, 109-116 (2003).

13. Papazoglou MP and Georgakopoulos D: 'Service-Oriented Computing', Communications of the ACM, 46, No 10, pp $24-28$ (2003).

14. Thint M, Case S and Azvine B: 'Soft Computing and Personal Information Provision', Enhancing the Power of the Internet, Ed. Nikravesh M et al., Springer (2003).

15. Chou J and Simerly T: 'How DLNA and UPnP will enable easy home video networks', Streaming Media DSP Group Texas Instruments Inc. http://www.videsignline.com/howto/showArticle.jhtml?arti cleId=170701703\&pgno=2

16. Nguyen D et al, Delivering Services by Building and Running Virtual Organisations, BT Technol J, 24 No 1, pp 141 - 152 (2006).

17. Nissen ME: 'Beyond electronic disintermediation through multi-agent systems', Logistics Information Management, 14 No 4, pp 256 - 275 (2001).

18. Li Y et al, 'Self Service Reservation in the Fieldforce', BT Technol J, 24 No 1, pp 40 - 47 (2006).

19. Jinmin $\mathrm{Hu}$, Grefen $\mathrm{P}$ (Dept. of Computer Science, Twente University, Enschede, Netherlands): 'Component Based System Framework for Dynamic B2B Interaction', Proceedings of 26th Annual International Computer Software and Applications, 26-29 Aug. 2002, IEEE Computer Soc, pp 557 - 62 (2002). 